\title{
ASSOCIATION OF POLYCYSTIC OVARY SYNDROME AND A NON-DIPPING BLOOD PRESSURE PATTERN IN YOUNG WOMEN
}

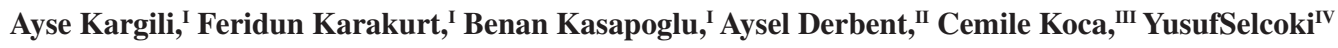

doi: $10.1590 /$ S1807-59322010000500004

Kargili A, Karakurt F, Kasapoglu B, Derbent A, Koca C, Selcoki Y. Association of polycystic ovary syndrome and a nondipping blood pressure pattern in young women. Clinics. 2010;65(5):475-9.

OBJECTIVE: The association between polycystic ovarian syndrome and increased cardiovascular disease risk is still a controversial issue. In light of data documenting some common pathways or common end-points, the present study was undertaken to determine whether there is a relationship between sleep blood pressure pattern disturbances and polycystic ovarian syndrome in young women. METHOD: The daytime and nighttime ambulatory blood pressures (BPs) were determined for each subject, according to the actual waking and sleeping times recorded in their individual diaries, in this cross-sectional study.

RESULTS: The study group comprised 168 women (mean age: $25.7 \pm 5.5$ ) diagnosed with polycystic ovarian syndrome, while the control group included 52 age- and BMI-matched healthy subjects (mean age: 26.1 \pm 5.4 ). When nocturnal BP declines very little or not at all, with the BP falling less than $10 \%$ during sleep compared with waking values, this pattern is classified as a non-dipping BP pattern. However, the non-dipping pattern of BP changes was significantly more common in polycystic ovarian syndrome patients compared to the control group ( $\mathrm{p}<0.01)$. The prevalence of a non-dipping BP pattern was $43.4 \%$ (73 patients) in polycystic ovarian syndrome patients and $3.9 \%$ (2 patients) in the control group.

CONCLUSION: Our cross-sectional study revealed that a non-dipping BP pattern is highly prevalent in polycystic ovarian syndrome patients, even if they are young and non-obese.

KEYWORDS: Polycystic ovary syndrome; non-dipping blood pressure.

\section{INTRODUCTION}

Polycystic ovarian syndrome (PCOS), which affects $5-10 \%$ of women of reproductive age, has some characteristic clinical features, including amenorrhea, oligomenorrhea or other irregular uterine bleeding, hirsutism, acne, and/or obesity. ${ }^{1,2}$ Unfortunately, patients with PCOS have an increased risk for type 2 diabetes mellitus,

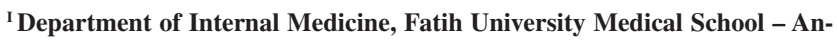
kara, Turkey.

II Department of Obstetrics and Gynecology, Fatih University Medical School - Ankara, Turkey.

III Department of Biochemistry, Fatih University Medical School - Ankara, Turkey.

IV Department of Cardiology, Fatih University Medical School - Ankara, Turkey.

E-mail: benankasapoglu@hotmail.com

Tel.: + 90-0312-4829166 or 2126262

Received for publication on October 30, 2009

First review completed on December 01, 2009

Accepted for publication February 17, 2010
}

endometrial carcinoma, and spontaneous abortions. ${ }^{3,4}$ Moreover, a clear association between PCOS and increased cardiovascular disease has been reported in many recent studies. ${ }^{5}$

A 3.3-fold higher risk of cardiovascular death or myocardial infarction in postmenopausal women with clinical features of PCOS has been reported recently. ${ }^{6}$ The suspected link between PCOS and cardiovascular disease is insulin resistance, which plays a central role not only in PCOS development but also in metabolic syndrome, a common syndrome that constitutes a combination of disturbances of carbohydrate and lipid metabolism, increased waist circumference, and hypertension. In addition to impaired endothelial-dependent vasodilatation, both increased carotid intima media wall thickness and amplified pulse-wave velocity have been established in PCOS as early clinical markers of atherosclerosis. ${ }^{7-9}$ Moreover, several markers of cardiovascular risk, such as tumor necrosis factor-, homocysteine, plasminogen activator inhibitor-1, 
and highly sensitive C-reactive protein (hsCRP), are increased in women with PCOS. ${ }^{10,11}$

When nocturnal blood pressure (BP) declines very little or not at all, with the BP falling less than 10\% during sleep compared with waking values, this pattern is classified as a non-dipping BP pattern. Because non-dippers have an elevated risk of target organ damage and increased incidence of fatal and non-fatal cardiovascular events compared to dippers, much attention has been given to the 24-hour BP patterns. ${ }^{12-13}$ Although the exact mechanisms are still not clear, endothelial impairment and oxidative stress may contribute to the non-dipping BP pattern, autonomic dysfunction, obesity, and insulin resistance. ${ }^{14-16}$

In light of the data documenting some common pathways or common end-points for non-dipping BP pattern and PCOS, the present study was undertaken to determine whether there is a relationship between sleep BP pattern disturbances and PCOS in young women.

\section{METHODS}

\section{Study design}

The study was carried out at Fatih University Hospital. A cross-sectional study design was used to compare the prevalence of a non-dipping BP pattern in healthy women and women with PCOS. The patients and controls were individually matched for age and body mass index (BMI).

This study was approved by the Fatih University School of Medicine Ethics Committee and was conducted in accordance with the ethical principles described by the Declaration of Helsinki. Written informed consent was obtained from all participants.

\section{Subjects}

Our study included consecutive outpatients admitted to the Department of Endocrinology of Fatih University, Turkey between January to July 2009 and who were diagnosed with PCOS. Age- and BMI-matched subjects were used as the control group.

The diagnosis of PCOS was based on the revised 2003 consensus on diagnostic criteria and long-term health risks related to PCOS published by the Rotterdam ESHRE/ ASRM-sponsored PCOS consensus workshop group. ${ }^{17}$ All diagnoses were made by an experienced ultrasonographer.

The exclusion criteria used when selecting patients and controls were as follows: pregnancy; hypothyroidism; hyperprolactinemia; Cushing's syndrome; non-classical congenital adrenal hyperplasia; current or previous (within the last 6 months) use of oral contraceptives, glucocorticoids, anti-androgens, ovulation induction agents, other hormones, or anti-diabetic/anti-obesity drugs; patients with hyperandrogenism alone; history of any neoplasia; and any type of kidney disease or use of anti-hypertensive drugs.

A complete physical examination was performed on all subjects, including measurement of height, weight, heart rate, and systolic (SBP) and diastolic (DBP) blood pressure by standard methods. The BMI was calculated as weight in $\mathrm{kg} / \mathrm{height}$ in square meters for all subjects. Waist circumference, taken midway between the lowest rib and the iliac crest, and hip circumference, taken at the level of the greater trochanters, was measured to the nearest millimeter using a flexible tape.

\section{Blood samples}

All blood samples were taken in the morning after a 12-h fast. Serum samples were stored at $-80^{\circ} \mathrm{C}$ until the analysis date. Fasting blood glucose, serum C-reactive protein (CRP), uric acid, alanine amino transferase (ALT), aspartate amino transferase (AST), gamma-glutamyl transferase (GGT), alkaline phosphatase (ALP), total cholesterol, high-density lipoprotein cholesterol (HDL-C), low-density lipoprotein cholesterol (LDL-C), triglycerides, follicule stimulating hormone (FSH), luteinizing hormone (LH), and insulin levels were measured.

The homeostasis model assessment of insulin resistance (HOMA-IR) was calculated by the following formula (18):

HOMA-IR= fasting insulin $(\mathrm{mU} / \mathrm{ml}) \times$ fasting glucose $(\mathrm{mmol} / \mathrm{l}) / 22.5$.

\section{Ambulatory BP monitoring}

Ambulatory BP monitoring was obtained from every subject with a portable, automated noninvasive monitoring device (Space Labs 90207; Spacelabs Inc, Redmond, Washington). An appropriately sized ambulatory BP measurement (ABPM) cuff was placed on the participant's non-dominant arm, and two readings were manually initiated. Readings were repeated if the SBP or DBP fell outside the predefined acceptable ranges (SBP: 70-240 mmHg; DBP: $40-150 \mathrm{mmHg}$; and heart rate: $20-150$ beats/ min). During BP recordings, participants were instructed to keep their arms still and in an extended position. The monitors were programmed to record BP every 30 minutes throughout the 24-hour period.

The periods of daytime and nighttime were determined based on the actual waking and sleep times recorded in the subjects' individual diaries. The average BP during daytime and nighttime was calculated. Subjects were defined as "dippers" when their nighttime SBP fell by 
$10 \%$ or more compared to the daytime SBP. Likewise, subjects were defined as non-dippers when their SBP fell by $<10 \%$ compared to daytime SBP or rose above daytime SBP(reverse dipping).

\section{Statistical analysis}

The data were analyzed using the Student's t-test to determine whether differences were significant. $\mathrm{P}<0.05$ was considered statistically significant. All calculations were carried out with SPSS version 13 (SPSS, Chicago, IL).

\section{RESULTS}

The study group was composed of 168 women (mean age: $25.7 \pm 5.5$ ) who were diagnosed with PCOS, while the control group included 52 age- and BMI-matched healthy

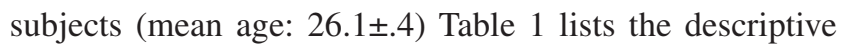
data of patients and controls.

Table 1 - Descriptive data of the study group and healthy control subjects.

\begin{tabular}{|c|c|c|c|}
\hline Variable & $\begin{array}{l}\text { PCOS } \\
\text { (n: } 168)\end{array}$ & $\begin{array}{l}\text { Control } \\
(\mathrm{n}: 52)\end{array}$ & $\mathrm{P}$ value \\
\hline Age (yr) & $25.7 \pm 5.5$ & $26.1 \pm 5.4$ & $>0.05$ \\
\hline Waist circumference $(\mathrm{cm})$ & $90.6 \pm 12.8$ & $78.5 \pm 9.3$ & 0.02 \\
\hline $\mathrm{BMI}\left(\mathrm{kg} / \mathrm{m}^{2}\right)$ & $26.8 \pm 3.4$ & $25.4 \pm 2.8$ & $>0.05$ \\
\hline ALT (units/l) & $18.5 \pm 6.7$ & $17.1 \pm 5.4$ & $>0.05$ \\
\hline AST (units/l) & $17.3 \pm 6.5$ & $16.3 \pm 5.6$ & $>0.05$ \\
\hline GGT (units/l) & $17.2 \pm 7.4$ & $16.4 \pm 6.2$ & $>0.05$ \\
\hline ALP (units/l) & $66.1 \pm 13.4$ & $55.7 \pm 10.4$ & $>0.05$ \\
\hline Triglyceride (mg/dl) & $90.9 \pm 28.2$ & $89.0 \pm 22.5$ & $>0.05$ \\
\hline Total cholesterol (mg/dl) & $168.7 \pm 36.7$ & $162.7 \pm 32.2$ & $>0.05$ \\
\hline HDL-C (mg/dl) & $55.5 \pm 9.7$ & $54.7 \pm 7.4$ & $>0.05$ \\
\hline LDL-C (mg/dl) & $91.9 \pm 22.3$ & $91.2 \pm 26.7$ & $>0.05$ \\
\hline $\mathrm{CRP}(\mathrm{mg} / \mathrm{l})$ & $5.6 \pm 3.8$ & $5.1 \pm 2.7$ & $>0.05$ \\
\hline Urea $(\mathrm{mg} / \mathrm{dl})$ & $24.8 \pm 4.7$ & $23.9 \pm 5.4$ & $>0.05$ \\
\hline Creatinine $(\mathrm{mg} / \mathrm{dl})$ & $0.61 \pm 0.1$ & $0.59 \pm 0.1$ & $>0.05$ \\
\hline HOMA-IR & $2.5 \pm 1.4$ & $1.3 \pm 0.9$ & 0.02 \\
\hline $\begin{array}{l}\text { Non-dipping status preva- } \\
\text { lence }\end{array}$ & $43.4 \%$ & $3.9 \%$ & $<0.01$ \\
\hline Daytime SBP & $113.8 \pm 10.8$ & $96.0 \pm 9.7$ & $<0.01$ \\
\hline Daytime DBP & $75.9 \pm 6.3$ & $66.3 \pm 6.5$ & 0.02 \\
\hline Nighttime SBP & $105.4 \pm 13.8$ & $88.0 \pm 2.3$ & $<0.01$ \\
\hline Nighttime DBP & $70.0 \pm 7.8$ & $57.2 \pm 5.4$ & 0.02 \\
\hline
\end{tabular}

Data are mean \pm SD, unless specified otherwise. BMI, body mass index; CRP, C-reactive protein; ALT, alanine aminotransferase; AST, aspartate aminotransferase GGT, gamma-glutamyltransferase; ALP, alkaline phosphatase; HDL-C, high-density lipoprotein cholesterol; LDL-C, low-density lipoprotein cholesterol; SBP, systolic blood pressure; DBP, diastolic blood pressure.
There was no statistically significant difference between PCOS patients and the control group with respect to the lipid profile, including total cholesterol, triglyceride, and HDL and LDL cholesterol levels. Although there was no statistically significant difference in fasting blood glucose levels between the two groups, as expected, the HOMA-IR values of the PCOS group were statistically significantly higher than the values of the control group ( 2.5 vs. 1.3, respectively, $\mathrm{p}=0.02$ ); waist circumference showed the same trend $(90.6 \pm 12.8 \mathrm{~cm}$ vs. $78.5 \pm 9.3 \mathrm{~cm}$, respectively, $\mathrm{p}=0.02)$.

Moreover, the non-dipping BP patterns were significantly more common in PCOS patients compared to the control group $(\mathrm{p}<0.01)$. The prevalence of a non-dipping BP pattern was $43.4 \%$ (73 patients) in PCOS patients and 3.9\% (2 patients) in the control group. The calculated power of the study was $99.2 \%$.

\section{DISCUSSION}

We found a significantly increased prevalence of a nondipping BP pattern in PCOS patients compared to age- and BMI-matched controls. Because the PCOS subjects and matching controls had similar BMI values (26.8 and 25.4, respectively), this increased prevalence of non-dipping $\mathrm{BP}$ changes cannot be attributed to the increased BMI. Similar to our results, Arslanian et al. demonstrated that adolescents with PCOS and impaired glucose tolerance lacked the normal nocturnal decline in BP. ${ }^{19}$

It is also interesting that there was no statistically significant difference between PCOS and control group patients with respect to the lipid profile, including total cholesterol, triglyceride, and HDL and LDL cholesterol levels, which were in the normal range in both groups. This finding may be ascribed to the young age of our study participants and their active life styles.

The increased risk of cardiovascular disease in patients with PCOS is still controversial because sufficient epidemiological data are not present. ${ }^{20,21}$ Moreover, 24-hour ambulatory SBP measurements revealed higher BP values in women with PCOS compared with controls, which may be evidence for the development of continual hypertension later in life. ${ }^{22}$ However, to the best of our knowledge, the non-dipping BP pattern has rarely been studied in PCOS patients. In contrast, the association of insulin resistance and non-dipping BP pattern has been investigated in few studies. In patients with metabolic syndrome, another consequence of insulin resistance, a decrease in the normal nocturnal BP decline, has been clearly defined. ${ }^{23}$

Although BMI and lipid profile were in the normal range, the prevalence of the non-dipping BP pattern was $43.4 \%$ (73 patients) in PCOS patients, which is a very high rate 
compared to normal population. Currently, the incidence of cardiovascular diseases not only increases non-dippers, but its prognosis also worsens. ${ }^{24-26}$ Gorostidi et al. found the presence of non-dipping BP pattern to be as high as $56.6 \%$ in a population with a high cardiovascular risk. ${ }^{27}$

A non-dipping BP pattern is regarded as an independent predictor of future cardiovascular events. ${ }^{28}$ Moreover, it is also associated with left ventricular hypertrophy and renal dysfunction. ${ }^{29,30}$ Because the main determinant of the circadian variations in BP appears to be the sympathetic nervous system, this system is suspected in the pathophysiology of the non-dipping BP pattern. ${ }^{31}$ Vascular endothelial dysfunction is another pathophysiological mechanism commonly suspected to play a role in the non-dipping blood pressure pattern. ${ }^{32}$ Whatever the underlying mechanisms, the diseases that predispose to a blunted nocturnal BP fall must be diagnosed earlier and follow-up is essential.

In some previous studies, insulin resistance was the most important feature of PCOS..$^{33,34}$ Supporting our data, a relationship between insulin resistance and a non-dipping BP pattern was previously demonstrated.$^{35}$ Furthermore, the cardiovascular risk in PCOS patients is correlated with the degree of hyperinsulinemia. ${ }^{36}$ Our findings further support these data based on the fact that non-dipping BP changes are more common in the PCOS group than controls. Although HOMA-IR values were statistically significantly different from the controls, the lipid profile and BMI were not.

Another very important and interesting finding of our study was that, although the BMI of the two groups were similar, there was a statistically significant difference between PCOS patients and the control group in regards to the waist circumference. Similarly, Dewailly et al. reported that waist circumference is one of the most reliable measurements of increased abdominal adipose tissue and positively correlates with insulinemia, insulin resistance, and both plasma luteinizing hormone and estradiol levels. ${ }^{37}$ Increased waist circumference is an independent risk factor for hypertension. ${ }^{38}$ Waist circumference is also important because it is one of the major components of metabolic syndrome, which is also clearly associated with increased cardiovascular disease risk.

In conclusion, our cross-sectional study revealed that a non-dipping BP pattern is highly prevalent in PCOS patients, even if they are young and non-obese. Although the crosssectional study design does not permit us to determine a cause-and-effect relationship and the mechanisms linking these two diseases are unclear, insulin resistance is the most suspected pathway. Moreover, the difference in the waist circumferences of the two groups may also play an additional role in BP changes. Further prospective studies are warranted not only to verify this relationship but also to elucidate the exact mechanisms.

\section{REFERENCES}

1. Zawadzki JK, Dunaif A. Diagnostic criteria for polycystic ovary syndrome: towards a rational approach. In: Dunaif A, Givens JR, Haseltine FP, Merriam GR, editors. Polycystic Ovary Syndrome. Oxford, England: Blackwell Scientific. 1992;377-84.

2. Azziz R, Woods KS, Reyna R, Key TJ, Knochenhauer ES, Yildiz BO. The prevalence and features of the polycystic ovary syndrome in an unselected population. J Clin Endocrinol Metab. 2004;89:2745-9.

3. Wang JX, Davies MJ, Norman RJ. Polycystic ovarian syndrome and the risk of spontaneous abortion following assisted reproductive technology treatment. Hum Reprod. 2001;16:2606-9.

4. Glueck CJ, Phillips H, Cameron D, Sieve-Smith L, Wang P. Continuing metformin through pregnancy in women with polycystic ovary syndrome appears to safely reduce first-trimester spontaneous abortion: a pilot study. Fertil Steril. 2001;75:46-52.

5. Cascella T, Palomba S, De Sio I, Manguso F, Giallauria F, De Simone $\mathrm{B}$, et al. Visceral fat is associated with cardiovascular risk in women with polycystic ovary syndrome. Hum Reprod. 2008;23:153-9.

6. Shaw LJ, Bairey Merz CN, Azziz R, Stanczyk FZ, Sopko G, Braunstein GD, et al. Postmenopausal women with a history of irregular menses and elevated androgen measurements at high risk for worsening cardiovascular event-free survival: results from the National Institutes of Health-National Heart, Lung, and Blood Institute sponsored Women's Ischemia Syndrome Evaluation. J Clin Endocrinol. Metab. 2008;93:1276-84.
7. Orio F Jr, Palomba S, Cascella T, De Simone B, Di Biase S, Russo $\mathrm{T}$, et al. Early impairment of endothelial structure and function in young normal-weight women with polycystic ovary syndrome. J Clin Endocrinol Metab. 2004;89:4588-93.

8. Talbott EO, Zborowski J V, Rager J R, Boudreaux MY, Edmundowicz DA, Guzick DS. Evidence for an association between metabolic cardiovascular syndrome and coronary and aortic calcification among women with polycystic ovary syndrome. J. Clin. Endocrinol. Metab. 2004;89:5454-61.

9. Diamanti-Kandarakis E, Alexandraki K, Piperi C, Protogerou A, Katsikis I, Paterakis T, et al. Inflammatory and endothelial markers in women with polycystic ovary syndrome. Eur J Clin Invest. 2006;36:691-7.

10. Loverro G, Lorusso F, Mei L, Depalo R, Cormio G, Selvaggi L, et al. Serum C-reactive protein in polycystic ovary syndrome 355 The plasma homocystein levels are increased in polycystic ovary syndrome. Gynecol Obstet Invest. 2002;53:157-62.

11. Guo L, Hu XS, Guo ZR, Kang GD, Wu M, Zhou H. [Association and interaction between the components of metabolic syndrome and cardiovascular disease]. Zhonghua Xin Xue Guan Bing Za Zhi. 2009;37:644-7.

12. Brotman DJ, Davidson MB, Boumitri M, Vidt DG. Impaired diurnal blood pressure variation and all-cause mortality. Am J Hypertens. 2008;21:92-7. 
13. Dolan E, Stanton A, Thijs L, Hinedi K, Atkins N, McClory S, et al. Superiority of ambulatory over clinic blood pressure measurement in predicting mortality: The Dublin outcome study. Hypertension. 2005;46:156-61.

14. Higashi Y, Nakagawa K, Kimura M, Noma K, Hara K, Sasaki S, et al. Circadian variation of blood pressure and endothelial function in patients with essential hypertension: a comparison of dippers and non-dippers. J Am Coll Cardiol. 2002;40:2039-43.

15. Kohara K, Nishida W, Maguchi M, Hiwada K. Autonomic nervous function in non-dipper essential hypertensive subjects: evaluation by power spectral analysis of heart rate variability. Hypertension. 1995;26:808-14.

16. Kotsis V, Stabouli S, Bouldin M, Low A, Toumanidis S, Zakopoulos N. Impact of obesity on 24-hour ambulatory blood pressure and hypertension. Hypertension. 2005;45:602-7.

17. The Rotterdam ESHRE/ASRM-Sponsored PCOS consensus workshop group. Revised 2003 consensus on diagnostic criteria and long-term health risks related to polycystic ovary syndrome (PCOS). Hum Reprod. 2004; 19:41-7.

18. Haffner SM, Miettinen H, Stern MP. The homeostasis model in the San Antonio heart study. Diabetes Care. 1997;20:1087-92.

19. Arslanian SA, Lewy VD, Danadian K. Glucose intolerance in obese adolescents with polycystic ovary syndrome: roles of insulin resistance and beta-cell dysfunction and risk of cardiovascular disease. J Clin Endocrinol Metab. 2001;86:66-71.

20. Amowitz LL, Sobel BE. Cardiovascular consequences of polycystic ovary syndrome. Endocrinol Metab Clin North Am. 1999;28:439-58.

21. Talbott E, Guzick D, Clerici A, Berga S, Detre K, Weimer K, et al. Coronary heart disease risk factors in women with polycystic ovary syndrome. Arterioscler Thromb Vasc Biol. 1995;15:821-6.

22. de Faire U, Lindvall K, Nilsson B. Noninvasive ambulatory $24 \mathrm{~h}$ blood pressures and basal blood pressures predict the development of sustained hypertension from a borderline state. Am J Hypertens. 1993;6:149-55.

23. Hermida RC, Chayán L, Ayala DE, Mojón A, Domínguez MJ, Fontao $\mathrm{MJ}$, et al. Association of metabolic syndrome and blood pressure nondipping profile in untreated hypertension. Am J Hypertens. 2009;22:307-13

24. Pickering TG. The clinical significance of diurnal blood pressure variations. Dippers and nondippers. Circulation. 1990;81:700-2.

25. Verdecchia P, Porcellati C, Schillaci G, Borgioni C, Ciucci A, Battistelli $\mathrm{M}$, et al. Ambulatory blood pressure variations and silent cerebrovascular damage in elderly patients with hypertension. J Hypertension. 1992; 10:875-8
26. Staessen JA, Asmar R, De Buyzere M, Imai Y, Parati G, Shimada K, et al. Task Force II: blood pressure measurement and cardiovascular outcome. Blood Press Monit. 2001;6:355-70.

27. Gorostidi M, Sobrino J, Segura J, Sierra C, de la Sierra A, Hernandez del Rey R, et al. Ambulatory blood pressure monitoring in hypertensive patients with high cardiovascular risk: a cross-sectional analysis of a 20 000-patient database in Spain. J Hypertens 2007; 25:977-84.

28. Lakka M, Laaksonen E, Lakka A, Niskanen K, Kompusalo E, Tuomilehto J, et al. The metabolic syndrome and total cardiovascular disease mortality in middle-aged men. J Am Med Assoc. 2002;288:270916.

29. Lind L, Andersson PE, Andern B, Hanni A, Lithell HO. Left ventricular hypertrophy in hypertension is associated with insulin-resistance metabolic syndrome. J Hypertens. 1995; 13:433-8.

30. Palaniappan L, Carnethon M, Fortmann SP. Association between microalbuminouria and the metabolic syndrome: NHANES III. Am J Hypertens. 2003;16:952-8.

31. Sica DA. What are the influences of salt, potassium, the sympathetic nervous system, and the renin-angiotensin system on the circadian variation in blood pressure? Blood Press Monit. 1999;4:9-16.

32. Ekmekci H, Ekmekci OB. The role of adiponectin in atherosclerosis and thrombosis. Clin Appl Thromb Hemost. 2006;12:163-8.

33. Mahabeer S, Jialal I, Norman RJ, Naidoo C, Reddi K, Joubert SM Insulin and $\mathrm{C}$-peptide secretion in non-obese patients with polycystic ovarian disease. Hormone Metab Res. 1989;21:502-6.

34. Robinson S, Kiddy D, Gelding SV, Willis D, Niththyananthan R, Bush A, et al. The relationship of insulin sensitivity to menstrual pattern in women hyperandrogenism and polycystic ovaries. Clin Endocrinol. $1993 ; 39: 351-5$

35. Nakano S, Kitazawa M, Tsuda S, Himeno M, Makiishi H, Nakagawa A, et al. Insulin resistance is associated with reduced nocturnal falls of blood pressure in normotensive, nonobese type 2 diabetic subjects. Clin Exp Hypertens. 2002;24:65-73.

36. Goodarzi MO, Korenman SG. The importance of insulin resistance in polycystic ovary syndrome. Fertil Steril. 2003;80:72-6

37. Dewailly D, Cortet-Rudelli C, Deroubaix-Allard D. Markers of abdominal adipose tissue in women: relationship to ovarian function. Trends Endocrinol Metab. 1998;9:68-71.

38. Gilardini L, Parati G, Sartorio A, Mazzilli G, Pontiggia B, Invitti C. Sympathoadrenergic and metabolic factors are involved in ambulatory blood pressure rise in childhood obesity. J Hum Hypertens. 2008;22:7582 . 